\title{
Smile Attractiveness Evaluation of Patients Selected for a U.S.-Based Board Certification Examination
}

\author{
Jen Soh ${ }^{1}$ Zheng Dong Wang $\quad$ Wei Bing Zhang ${ }^{2}$ Chung How Kau,๑
}

1Private Practice, Singapore

2Department of Orthodontics, Nanjing Medical University, Nanjing, Jiangsu, People's Republic of China

${ }^{3}$ Department of Orthodontics, The University of Alabama at

Birmingham, Birmingham, Alabama, United States

Eur J Dent 2021;15:630-638.

\begin{abstract}
Address for correspondence Chung How Kau, BDS, MScD, MBA, MOrth, PhD, FDSEdin, FFDIre(Ortho), FDSGlas, FAMS, FICD(Ortho), ABO, Department of Orthodontics, The University of Alabama at Birmingham, Suite 305, 1919 7th Ave South, Birmingham, AL 35294, United States (e-mail: ckau@uab.edu).
\end{abstract}

\begin{abstract}
Keywords

- board certification examination

- orthodontics

- perception

- smile attractiveness

Objectives The aims of this study were to assess smile attractiveness of a collection of 68 smiling photographs of successfully treated cases submitted to the American Board of Orthodontics $(\mathrm{ABO})$ clinical examination and identify variables that influence the assessment.

Materials and Methods A panel of 81 non-Caucasian assessors from various clinical disciplines were instructed to score the smile attractiveness on a visual analog scale from 1 (least attractive) to 10 (most attractive) and to select which components contributed to a lesser attractive smile. The mean, standard deviations (SDs), and quartiles of the smile attractiveness were obtained with descriptive statistics. Multilinear regression analysis was performed to investigate the scores of the perceived quality of smile attractiveness when the clinical disciplines and gender of the assessors were the factors taken into consideration. Receiver operating characteristic (ROC) curve was generated to establish the relationship between smile attractiveness and the achievement of a perfect smile.

Results The mean (SD) rating of each clinical photograph of the anterior occlusion on smiling ranged from 3.11 (1.47) as the least attractive smile to $7.59(1.45)$ as the most attractive smile. The overall mean (SD) score for smile attractiveness was 5.30 (1.10). Problems associated with teeth, gingiva, and lips corresponded with a reduction of the smile attractiveness score by $1.56,1.82$, and 1.47 , respectively. Gender was not associated with smile attractiveness ratings. Orthodontists, periodontists, and prosthodontists demonstrated no difference in the ratings, while plastic surgeons were more critical than orthodontists regarding smile attractiveness.

Conclusions This study suggested that only 2 out of $68 \mathrm{AOB}$ validated treatment finishes had a perfect and attractive smile.
\end{abstract}

\section{Introduction}

A major motivation of individuals who seek orthodontic treatment is their dissatisfaction with the dental appearance related to the malocclusion and a desire to have an attractive smile that reflects the visible treatment outcome that is of primary interest to both patients and clinicians. ${ }^{1}$ Studies have found that malocclusions were associated with psychosocial implications such as job prospect at interviews, ${ }^{2}$ the search for dating partners, ${ }^{3}$ self-esteem on orthodontic treatment need, ${ }^{4}$ and perceived intelligence. ${ }^{5}$ published online July 24, 2021
DOI https://doi.org/ $10.1055 / \mathrm{s}-0041-1726670$ ISSN $1305-7456$ (c) 2021. European Journal of Dentistry.

This is an open access article published by Thieme under the terms of the Creative Commons Attribution-NonDerivative-NonCommercial-License, permitting copying and reproduction so long as the original work is given appropriate credit. Contents may not be used for commercial purposes, or adapted, remixed, transformed or built upon. (https://creativecommons.org/licenses/by-nc-nd/4.0/).

Thieme Medical and Scientific Publishers Pvt. Ltd. A-12, 2nd Floor, Sector 2, Noida-201301 UP, India 
An attractive smile can be viewed as the end point of the clinical outcome that reflects the quality of the orthodontic treatment and its effects on smile esthetics perceived by the patients themselves, their peers, and dental professionals. ${ }^{6,7}$ Although the standard of an ideal smile remains undetermined, an attractive smile can be described as a "balanced" smile having a harmonious interrelationship between the teeth, gingiva, and lips interplaying within the dynamic display zone of the face. ${ }^{8}$ The anterior esthetic zone displayed on smiling is unique to an individual face and is framed by three components, namely, the dental, gingival, and soft tissue components. ${ }^{9-11}$ The dental component comprises the anterior teeth alignment, position, size, shape, and color. In the vertical plane, the social six comprising the upper incisal edges and canine tips set in harmony with reference to the lower lip curvature upon full smile and the parallelism of the upper central incisors to the facial midline are supposedly the desired features of an attractive smile. ${ }^{12}$ The anteroposterior position and upper incisor inclination that provide incisor guidance, anterior teeth display, and support of the upper lip were found to be the features that influence the smile attractiveness. ${ }^{13}$ The round shape incisors are mostly preferred over triangular and square incisors. ${ }^{14}$ The size of the lateral incisor relative to the adjacent anterior teeth in terms of crown size proportion and height ratio was found to be integral when designing the smile. ${ }^{15}$ The teeth color has been shown to play a significant impact on patients' perception of smile attractiveness. ${ }^{16}$

The gingival component is characterized by its color, texture, shape, and amount of gingival show when smiling. ${ }^{17}$ The amount of upper gingival show up to $3 \mathrm{~mm}$ on smiling was found to be the accepted norm for an attractive smile. ${ }^{18}$ Gingival zenith symmetry and the absence of black interdental triangular spaces were desirable traits in pleasing smiles. ${ }^{19}$

The soft tissue component comprises the lips at rest and on smiling thus representing the frame of the smile of each individual. ${ }^{6}$ The resting position of the lips is described by the lip position, profile, and thickness. A high lip position reveals maximum crown length of upper teeth and more than $2 \mathrm{~mm}$ of gingival show, while a low lip position exposes less than $70 \%$ of the upper central incisors. The dynamic position of the lips is described by the upper lip curtain, symmetry, and curvature on smiling that is dependent on neuromuscular factors. The variables that were found to influence the perceived smile attractiveness include anterior smile line, posterior smile line, and upper lip curve. ${ }^{20}$ The upper lip position and thickness were found to be influential on perceived smile attractiveness. ${ }^{21}$

Studies have found that the assessment and perception of an attractive smile varied between dental professionals and laypersons. A systematic review reported that tooth morphology such as the size and shape of incisor, dental midline discrepancy, gingival exposure, buccal corridors, occlusal canting, overbite, and diastema were influential on the perception of smile attractiveness assessed by laypersons. ${ }^{22}$ Orthodontists were found to be more critical of asymmetric maxillary incisor crown height discrepancies than laypersons and general dentists in the evaluation of smile attractiveness. The perceived smile attractiveness was affected by unilateral reduced crown width and unilateral gingival margin height asymmetry. ${ }^{18}$ Dental professionals and laypersons were found to have different threshold levels when evaluating anterior dental attractiveness associated with gingival margin height differences of maxillary central incisors and dental midline discrepancy. ${ }^{23}$ Differential perception of the influence of anteroposterior position of incisors on dental attractiveness was found between laypersons and orthodontists. ${ }^{24}$

The evaluation of orthodontic treatment outcome to reflect the excellence and quality of treatment that is quantifiable has led to the development of occlusal indices such as the Peer Assessment Rating index and Index of Complexity Outcome and Need to serve the intended purpose..$^{25,26}$ The American Board of Orthodontics objective grading system (ABO-OGS) was developed during the years between 1994 and 1998 to overcome the limitations of Andrew's six keys for normal occlusion that was technically difficult to achieve and measure the occlusal goals. The six keys also did not correlate with the levels of malocclusion complexity and difficulty to end point results. Moreover, ABO-OGS is an objective assessment tool developed by orthodontists for the purpose of conducting an objective board examination to evaluate the quality of orthodontic treatment outcome primarily based on the occlusal traits measured from study models. A balanced smile characterized by a harmonious smile arch with lesser gingival show were found to define attractive smiles of patients who were considered as successfully treated to the standards of the $\mathrm{ABO} .^{27}$ However, the defining factors of the ABO-OGS were found to have very weak correlations with the perceived smile attractiveness of patients at posttreatment, although it is well regarded as the standard objective tool of choice to evaluate orthodontic treatment outcomes. ${ }^{28}$

The aims of the present study were to (1) assess the smile attractiveness of patients successfully treated to ABO standards by non-Caucasian assessors of diverse clinical disciplines, (2) determine assessors' rating differences, and (3) identify the dental components that influence the rating outcomes.

\section{Materials and Methods}

The materials for the study were screened and obtained from the orthodontic archive of the Department of Orthodontics, University of Alabama at Birmingham. Ethical approval to conduct the study was granted by the Institutional Review Board of the University of Alabama at Birmingham. Only cases that had completed orthodontic treatment and submitted to the ABO examination in Saint Louis, Missouri, United States were included for the study.

These cases required smile photographs of orthodontic patients with availability from the American Board Cases archive of the Department of Orthodontics, University of Alabama at Birmingham. The eligibility status of the completed cases used for the present study was the requirement that all the cases were validated to have passed the $A B O$ clinical examination within the past 5 years of recruitment 
time. A study sample of 68 patient records was required to detect an effect with $85 \%$ power and a significance level of $p=0.05$ based on the sample size calculation of a similar study. ${ }^{29}$ The inclusion and exclusion criteria to obtain the study records were as follows.

\section{Inclusion Criteria}

1. Complete final posttreatment records of patients

2. Case submissions to the $A B O$ examinations conducted between 2013 and 2018 and validated cases that were successfully treated.

\section{Exclusion Criteria}

1. Smile photographs of poor quality

2. Final smile photographs that did not fulfill the $A B O$ standards

3. Photograph that could not be calibrated adequately.

The smiles were further categorized into three groups: attractive, neutral, and unattractive for grading. A standardized template of $16 \times 9$ inch was used to crop the photographs to obtain a proportionate area around the upper and lower lips region to control any confounding factors. All the cropped photographs were imported into Adobe Photoshop CS version 9.0 (Adobe Systems, San Jose, California, United States) and the interpupillary line was used as reference to align the photographs. The interpupillary was used as the $X$-axis and the picture cropped to ensure the lips fell into the framework. The vertical frameworks included the commissures of the lips (-Fig. 1). These photos were placed on a power point slide in a random fashion and projected on a computer laptop with high screen resolution.

The survey was conducted on a group of health professionals of non-Caucasian descent. These health professionals were made up of different oral health specialists which included orthodontists, oral surgeons, plastic surgeons, and dentists. The assessors were instructed to score the smile attractiveness by choosing a number on a visual analog scale of 1 (least attractive) to 10 (most attractive) and to assess the perceived perfection of each smile and state which of the components, namely, the teeth, gums, or lips, that had made the smile less attractive when assessing each of the smile photographs. Each assessor had a total of 30 minutes to complete the survey and they did not compare photos between one another.

The study sample comprised 81 non-Caucasian health professionals of Chinese descent with an age range

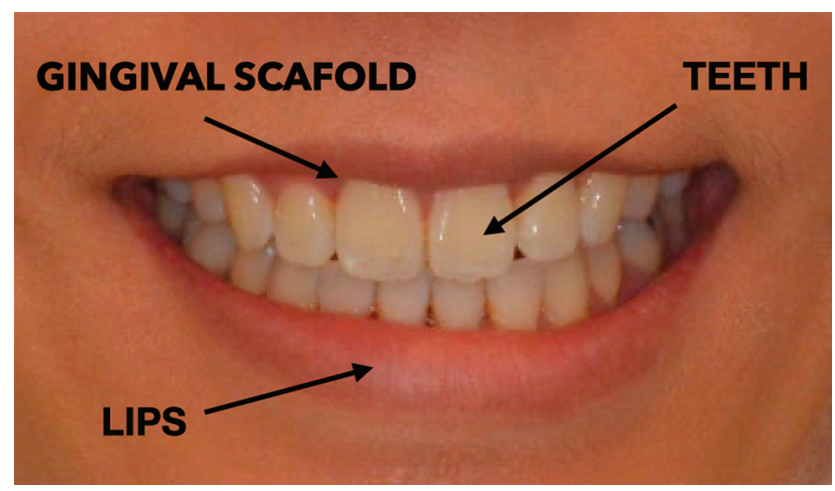

Fig. 1 Smile components demonstrating the teeth, gingival, and lips. between 20 and 40 years. The male and female assessors accounted for $27 \%$ and $73 \%$ of the study sample, respectively. The health professionals included 45 orthodontists (55\%), 19 periodontists (23.5\%), 14 plastic surgeons (17.3\%), and 3 prosthodontists (3.7\%).

\section{Statistical Methods}

Descriptive statistics were performed to determine the mean, standard deviations (SDs), and quartiles of the smile attractiveness. Additional considerations were given to the gender of the assessors and the professional clinical disciplines. Multilevel mixed linear regression (MLR) analysis was employed to analyze the influence of professional clinical disciplines and gender of the assessors on the smile attractiveness scores. The MLR analysis was also employed to analyze if the gingiva, lips, and teeth had influenced the final smile attractiveness outcomes. Finally, a receiver operating characteristic (ROC) curve was created to determine the threshold level between smile attractiveness and the perfect smile. All the statistical analyses were conducted using SAS 9.4 (Cary, North Carolina, United States).

\section{Results}

The results of the survey showed the following.

\section{Smile Attractiveness Ratings}

The smile attractiveness represented by the calculated mean and \pm SD of each patient was generated. The mean rating of each smile photograph ranged from $3.11 \pm 1.47$ (least attractive smile) to $7.59 \pm 1.45$ (most attractive smile). The overall mean for smile attractiveness was $5.30 \pm 1.10$. The distribution of ratings in percentiles is demonstrated in - Table $\mathbf{1}$.

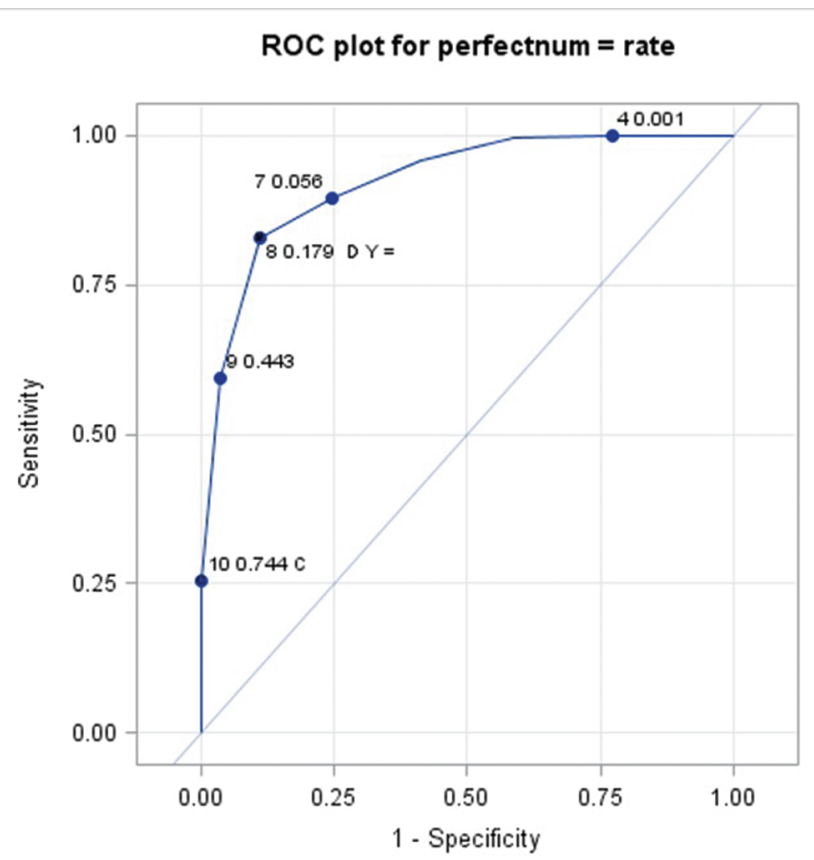

Fig. 2 Receiver operating characteristic (ROC) curve: a cutoff value of 7.4 for smile attractiveness rating demonstrating the best combined sensitivity and specificity values with the perfect smile. 
Smile Attractiveness of Patients for Certification Examination Soh et al. 633

Table 1 Distribution of ratings (0-10), perfect $(0,1)$, and problems with lips $(0,1)$, gums $(0,1)$, or teeth $(0,1)$ by patient ID

\begin{tabular}{|c|c|c|c|c|c|c|c|c|c|c|}
\hline \multirow[t]{2}{*}{ Patient ID } & \multicolumn{2}{|l|}{ Rate } & \multicolumn{2}{|c|}{ Perfect } & \multicolumn{2}{|c|}{ Problem with lips } & \multicolumn{2}{|c|}{ Problem with gums } & \multicolumn{2}{|c|}{ Problem with teeth } \\
\hline & Mean & SD & Mean & SD & Mean & SD & Mean & SD & Mean & SD \\
\hline 1 & 6.62 & 1.9 & 0.07 & 0.26 & 0.2 & 0.4 & 0.41 & 0.49 & 0.52 & 0.5 \\
\hline 2 & 7.14 & 1.44 & 0.16 & 0.37 & 0.73 & 0.45 & 0.02 & 0.16 & 0.12 & 0.33 \\
\hline 3 & 4.17 & 1.56 & 0.01 & 0.11 & 0.86 & 0.34 & 0.06 & 0.24 & 0.33 & 0.47 \\
\hline 4 & 7.09 & 1.72 & 0.28 & 0.45 & 0.27 & 0.45 & 0.1 & 0.3 & 0.37 & 0.49 \\
\hline 5 & 4.64 & 1.38 & 0 & 0 & 0.69 & 0.46 & 0.43 & 0.5 & 0.3 & 0.46 \\
\hline 6 & 4.98 & 1.56 & 0.01 & 0.11 & 0.4 & 0.49 & 0.41 & 0.49 & 0.51 & 0.5 \\
\hline 7 & 3.69 & 1.55 & 0 & 0 & 0.26 & 0.44 & 0.94 & 0.24 & 0.14 & 0.34 \\
\hline 8 & 3.11 & 1.47 & 0 & 0 & 0.14 & 0.34 & 0.38 & 0.49 & 0.93 & 0.26 \\
\hline 9 & 6.14 & 1.68 & 0.09 & 0.28 & 0.73 & 0.45 & 0.07 & 0.26 & 0.16 & 0.37 \\
\hline 10 & 5.73 & 1.8 & 0.06 & 0.24 & 0.3 & 0.46 & 0.23 & 0.43 & 0.58 & 0.5 \\
\hline 11 & 4.35 & 1.5 & 0 & 0 & 0.22 & 0.42 & 0.91 & 0.28 & 0.16 & 0.37 \\
\hline 12 & 4.16 & 1.57 & 0 & 0 & 0.31 & 0.46 & 0.89 & 0.32 & 0.23 & 0.43 \\
\hline 13 & 6.3 & 1.75 & 0.12 & 0.33 & 0.46 & 0.5 & 0.21 & 0.41 & 0.28 & 0.45 \\
\hline 14 & 5.63 & 1.92 & 0.06 & 0.24 & 0.4 & 0.49 & 0.21 & 0.41 & 0.44 & 0.5 \\
\hline 15 & 3.8 & 1.6 & 0 & 0 & 0.41 & 0.49 & 0.28 & 0.45 & 0.81 & 0.39 \\
\hline 16 & 4.7 & 1.61 & 0 & 0 & 0.53 & 0.5 & 0.32 & 0.47 & 0.44 & 0.5 \\
\hline 17 & 4.1 & 1.66 & 0.01 & 0.11 & 0.33 & 0.47 & 0.48 & 0.5 & 0.54 & 0.5 \\
\hline 18 & 5.1 & 1.73 & 0.02 & 0.16 & 0.44 & 0.5 & 0.3 & 0.46 & 0.42 & 0.5 \\
\hline 19 & 4.9 & 2.12 & 0.05 & 0.22 & 0.3 & 0.46 & 0.37 & 0.49 & 0.49 & 0.5 \\
\hline 20 & 4.48 & 1.62 & 0.01 & 0.11 & 0.54 & 0.5 & 0.04 & 0.19 & 0.64 & 0.48 \\
\hline 21 & 5.23 & 1.55 & 0.02 & 0.16 & 0.77 & 0.43 & 0.06 & 0.24 & 0.27 & 0.45 \\
\hline 22 & 4.68 & 1.46 & 0 & 0 & 0.26 & 0.44 & 0.83 & 0.38 & 0.17 & 0.38 \\
\hline 23 & 4.02 & 1.67 & 0 & 0 & 0.14 & 0.35 & 0.69 & 0.47 & 0.66 & 0.48 \\
\hline 24 & 6.78 & 1.94 & 0.25 & 0.43 & 0.26 & 0.44 & 0.09 & 0.28 & 0.44 & 0.5 \\
\hline 25 & 4.32 & 1.66 & 0.01 & 0.11 & 0.75 & 0.43 & 0.42 & 0.5 & 0.07 & 0.26 \\
\hline 26 & 4.41 & 1.59 & 0.03 & 0.16 & 0.34 & 0.48 & 0.61 & 0.49 & 0.45 & 0.5 \\
\hline 27 & 4.01 & 1.69 & 0 & 0 & 0.21 & 0.41 & 0.16 & 0.37 & 0.9 & 0.3 \\
\hline 28 & 7.27 & 1.7 & 0.36 & 0.48 & 0.2 & 0.4 & 0.14 & 0.34 & 0.33 & 0.47 \\
\hline 29 & 3.77 & 1.59 & 0 & 0 & 0.19 & 0.39 & 0.94 & 0.24 & 0.09 & 0.28 \\
\hline 30 & 6.17 & 1.74 & 0.04 & 0.19 & 0.59 & 0.49 & 0.04 & 0.19 & 0.48 & 0.5 \\
\hline 31 & 4.85 & 1.97 & 0 & 0 & 0.28 & 0.45 & 0.42 & 0.5 & 0.63 & 0.49 \\
\hline 32 & 4.89 & 1.7 & 0.01 & 0.11 & 0.43 & 0.5 & 0.46 & 0.5 & 0.31 & 0.46 \\
\hline 33 & 6.07 & 1.52 & 0.04 & 0.19 & 0.58 & 0.5 & 0.23 & 0.43 & 0.38 & 0.49 \\
\hline 34 & 4.93 & 1.7 & 0.02 & 0.16 & 0.25 & 0.43 & 0.83 & 0.38 & 0.12 & 0.33 \\
\hline 35 & 6.05 & 1.99 & 0.11 & 0.32 & 0.35 & 0.48 & 0.28 & 0.45 & 0.4 & 0.49 \\
\hline 36 & 5.63 & 1.81 & 0.05 & 0.22 & 0.54 & 0.5 & 0.41 & 0.49 & 0.11 & 0.32 \\
\hline 37 & 7.58 & 1.52 & 0.23 & 0.43 & 0.21 & 0.41 & 0.41 & 0.49 & 0.16 & 0.37 \\
\hline 38 & 4.59 & 1.72 & 0.01 & 0.11 & 0.58 & 0.5 & 0.54 & 0.5 & 0.19 & 0.39 \\
\hline 39 & 6.11 & 2.09 & 0.11 & 0.32 & 0.31 & 0.46 & 0.57 & 0.5 & 0.11 & 0.32 \\
\hline 40 & 5 & 1.65 & 0 & 0 & 0.93 & 0.26 & 0.05 & 0.22 & 0.17 & 0.38 \\
\hline 41 & 5.77 & 1.61 & 0.04 & 0.19 & 0.77 & 0.43 & 0.15 & 0.36 & 0.14 & 0.34 \\
\hline 42 & 7.35 & 1.73 & 0.28 & 0.45 & 0.27 & 0.45 & 0.36 & 0.48 & 0.12 & 0.33 \\
\hline 43 & 5.41 & 1.61 & 0.01 & 0.11 & 0.26 & 0.44 & 0.79 & 0.41 & 0.17 & 0.38 \\
\hline 44 & 4.96 & 1.87 & 0.05 & 0.22 & 0.73 & 0.45 & 0.12 & 0.33 & 0.22 & 0.42 \\
\hline 45 & 4.02 & 1.41 & 0 & 0 & 0.37 & 0.49 & 0.65 & 0.48 & 0.4 & 0.49 \\
\hline
\end{tabular}


Table 1 (Continued)

\begin{tabular}{|c|c|c|c|c|c|c|c|c|c|c|}
\hline \multirow[t]{2}{*}{ Patient ID } & \multicolumn{2}{|l|}{ Rate } & \multicolumn{2}{|c|}{ Perfect } & \multicolumn{2}{|c|}{ Problem with lips } & \multicolumn{2}{|c|}{ Problem with gums } & \multicolumn{2}{|c|}{ Problem with teeth } \\
\hline & Mean & SD & Mean & SD & Mean & SD & Mean & SD & Mean & SD \\
\hline 46 & 6.06 & 2.2 & 0.11 & 0.32 & 0.19 & 0.39 & 0.12 & 0.33 & 0.69 & 0.46 \\
\hline 47 & 5.31 & 1.48 & 0.03 & 0.16 & 0.56 & 0.5 & 0.01 & 0.11 & 0.53 & 0.5 \\
\hline 48 & 5.42 & 1.83 & 0.01 & 0.11 & 0.36 & 0.48 & 0.57 & 0.5 & 0.32 & 0.47 \\
\hline 49 & 3.27 & 1.77 & 0 & 0 & 0.37 & 0.49 & 0.67 & 0.47 & 0.46 & 0.5 \\
\hline 50 & 5.41 & 1.71 & 0.04 & 0.19 & 0.69 & 0.46 & 0.06 & 0.24 & 0.31 & 0.46 \\
\hline 51 & 5.38 & 2.19 & 0.06 & 0.24 & 0.36 & 0.48 & 0.67 & 0.47 & 0.1 & 0.3 \\
\hline 52 & 4.7 & 1.68 & 0.01 & 0.11 & 0.26 & 0.44 & 0.64 & 0.48 & 0.57 & 0.5 \\
\hline 53 & 5.89 & 1.57 & 0.05 & 0.22 & 0.44 & 0.5 & 0.22 & 0.42 & 0.42 & 0.5 \\
\hline 54 & 5.12 & 1.73 & 0.01 & 0.11 & 0.27 & 0.45 & 0.35 & 0.48 & 0.64 & 0.48 \\
\hline 55 & 5.17 & 1.68 & 0.01 & 0.11 & 0.77 & 0.43 & 0.11 & 0.32 & 0.23 & 0.43 \\
\hline 56 & 5.51 & 2.05 & 0.11 & 0.32 & 0.36 & 0.48 & 0.27 & 0.45 & 0.38 & 0.49 \\
\hline 57 & 6.35 & 2.05 & 0.13 & 0.33 & 0.58 & 0.5 & 0.14 & 0.35 & 0.34 & 0.48 \\
\hline 58 & 5.26 & 2.11 & 0.12 & 0.33 & 0.36 & 0.48 & 0.41 & 0.49 & 0.35 & 0.48 \\
\hline 59 & 6.36 & 1.89 & 0.06 & 0.24 & 0.39 & 0.49 & 0.09 & 0.28 & 0.55 & 0.5 \\
\hline 60 & 4.86 & 1.61 & 0 & 0 & 0.46 & 0.5 & 0.62 & 0.49 & 0.32 & 0.47 \\
\hline 61 & 5.81 & 1.59 & 0.04 & 0.19 & 0.46 & 0.5 & 0.33 & 0.47 & 0.36 & 0.48 \\
\hline 62 & 5.91 & 1.59 & 0.02 & 0.16 & 0.64 & 0.48 & 0.16 & 0.37 & 0.4 & 0.49 \\
\hline 63 & 7.59 & 1.45 & 0.41 & 0.5 & 0.36 & 0.48 & 0.13 & 0.33 & 0.15 & 0.36 \\
\hline 64 & 6.44 & 1.72 & 0.09 & 0.28 & 0.14 & 0.34 & 0.41 & 0.49 & 0.58 & 0.5 \\
\hline 65 & 4.33 & 1.44 & 0 & 0 & 0.27 & 0.45 & 0.93 & 0.26 & 0.11 & 0.32 \\
\hline 66 & 3.19 & 1.61 & 0 & 0 & 0.31 & 0.46 & 0.78 & 0.42 & 0.52 & 0.5 \\
\hline 67 & 7.33 & 1.6 & 0.27 & 0.45 & 0.63 & 0.49 & 0.01 & 0.11 & 0.11 & 0.32 \\
\hline 68 & 4.8 & 1.41 & 0 & 0 & 0.41 & 0.49 & 0.07 & 0.26 & 0.78 & 0.42 \\
\hline Total & 5.30 & 1.10 & 0.06 & 0.09 & 0.42 & 0.20 & 0.37 & 0.27 & 0.37 & 0.21 \\
\hline
\end{tabular}

Abbreviation: SD, standard deviation.

Note: Distribution of smile ratings for the total sample obtained from the visual analog scale. Quantile value minimum 3.11 25\% 4.54 Median $5.2075 \%$. 6.07 maximum 7.69 .

\section{Smile Attractiveness Perfection and Related Problems Affecting Rating Outcomes}

The assessors' ratings were affected by the problems with the teeth in $94 \%$ of the responses, $50 \%$ in the gingival, and $12 \%$ in the lips.

With the determinant of the lips as the primary factor for the smile outcome, the least problematic score $(4 \%$ of assessors) had an attractiveness score of $7.58 \pm 1.63$ and the most problematic score (75\% of assessors) had an attractiveness score of $5.08 \pm 1$. 88 . When the gums were taken as the determinant being the primary factor for the smile outcome, the least problematic score ( $2 \%$ of assessors) had an attractiveness score of $8.12 \pm 1.42$ and the most problematic score (96\% of assessors) had an attractiveness score of $4.69 \pm 1.98$. Finally, when the teeth were the determinant as the highest factor for the smile outcome, the least problematic score ( $8 \%$ of assessors) had an attractiveness score of 8.46 \pm 1.59 and the most problematic score ( $94 \%$ of assessors) had an attractiveness score of $3.42 \pm 1.83$. An interesting finding to note was that the most attractive and least attractive smile scores corresponded to the assessor's ratings of the teeth.

\section{Multilevel Mixed Linear Regression Analysis of Smile Attractiveness-Related Problems}

Multilevel mixed linear regression analysis was performed to predict the rating of the smile attractiveness being influenced by the lips, gums, teeth, occupation, and gender. - Table 2 represents the predictors of attractiveness obtained from MLR. The predications highlighted the lips, gums, and teeth as variables that affected the smile attractiveness. Of the assessors, only the plastic surgeons had a significant effect on the outcome.

\section{Receiver Operating Characteristic Curve}

ROC curve analysis is used in clinical epidemiology to quantify how accurately medical diagnostic tests (or systems) can discriminate between two patient states, typically referred to as "diseased" and "nondiseased." ${ }^{30}$ An ROC curve is based on the notion of a "separator" scale, on which results for the diseased and nondiseased form a pair of overlapping distributions. ${ }^{31}$ The complete separation of the two underlying distributions implies a perfectly discriminating test while complete overlap implies no discrimination. In our study, we 
Table 2 Predictors of attractiveness identified by multilevel mixed linear regression model

\begin{tabular}{|l|l|l|}
\hline Parameter & $\begin{array}{l}\text { Changes in } \\
\text { rating }(95 \% \mathrm{Cl})\end{array}$ & $\operatorname{Pr}>|t|$ \\
\hline Problems with lips & $1.47(1.56,1.37)$ & $<0.0001$ \\
\hline Problems with gums & $1.82(1.92,1.73)$ & $<0.0001$ \\
\hline Problems with teeth & $1.56(1.66,1.47)$ & $<0.0001$ \\
\hline $\begin{array}{l}\text { Periodontist vs. } \\
\text { orthodontist }\end{array}$ & $0.33(0.87,0.20)$ & 0.2252 \\
\hline $\begin{array}{l}\text { Plastic surgeon vs. } \\
\text { orthodontist }\end{array}$ & $1.98(2.60,1.37)$ & $<0.0001$ \\
\hline $\begin{array}{l}\text { Prosthodontist vs. } \\
\text { orthodontist }\end{array}$ & $0.02(1.15,1.19)$ & 0.9721 \\
\hline Female vs. male & $0.16(0.34,0.67)$ & 0.5341 \\
\hline
\end{tabular}

Abbreviation: SD, standard deviation.

Note: Model parameter changes in rating $(95 \% \mathrm{Cl}) \mathrm{Pr}>|t|$. Problems with lips $1.47(1.56,1.37)<0.0001$, problems with gingiva $1.82(1.92$, $1.73)<0.0001$, problems with teeth $1.56(1.66,1.47)<0.0001$, periodontist versus orthodontist $0.33(0.87,0.20) 0.2252$, plastic surgeon versus orthodontist $1.98(2.60,1.37)<0.0001$, prosthodontist versus orthodontist $0.02(1.15,1.19) 0.9721$, female versus male $0.16(0.34$ $0.67) 0.5341$.

used the ROC to determine two states, smile attractiveness versus a perfect smile.

This relationship was investigated with the use of sensitivity and specificity calculation to generate the ROC curve (-Fig. 2). A cutoff value of 7.4 for smile attractiveness rating was found to be the best representation of the combined sensitivity and specificity with the perfect smile. This meant that a cutoff value of 7.4 indicated the smallest difference between sensitivity and specificity among the variables. A review of the total sample of smile attractiveness ratings found that only 2 out of 68 cases or $2.94 \%$ of the study sample were represented in this sample ( $\mathbf{- F i g . ~ 2 ) . ~}$

\section{Discussion}

An interesting aspect of the present study was the assessment of smile attractiveness of orthodontic patients finished to $\mathrm{ABO}$ standards of quality by non-Caucasian assessors. The non-Caucasian health professionals were more critical when assessing smile attractiveness according to their best and worst smile ratings (most attractive: $7.59 \pm 1.45$; least attractive: $3.11 \pm 1.47$ ) when compared with the Caucasian health professionals (most attractive: $8.46 \pm 1.59$; least attractive: $3.42 \pm 1.83$ ) who evaluated the same set of 68 pictures. ${ }^{29}$ This observation was a direct comparison of the maximum and minimum scores without performing a statistical evaluation. Thus, whether the difference in the clinical assessment of smile attractiveness was statistically significant between non-Caucasian and Caucasian health professionals remains to be determined. The results suggested that there was no universal agreement to a "perfect smile" according to the ratings obtained from the pictures of the finished orthodontic cases that satisfied the ABO standards. The present study suggested that cases treated to good intra- and interarch occlusal relationships at static occlusion as measured from the study casts according to ABO-OGS standards did not necessarily translate into the equivalent of a good smile attractiveness that was delivered to the patients. The present and previously published studies were consistent with the finding that achieving a perfect smile proved to be elusive despite having achieved a good occlusion. The ABO-OGS by itself is a well-established professionally developed objective tool that serves the purpose of assessing the quality of orthodontic finishing outcome of completed orthodontic cases based on the occlusion. It allows board examiners to have a common communicating platform to deliberate and determine the level of details demonstrated by the biomechanical skills of prospective candidates according to the finished occlusion. It is still the best available and reliable measuring tool to assess and establish the outcome of the clinical and radiographic section of the board examination. It is without doubt that ABO-OGS is appropriately stringent to measure the occlusal traits of the finished occlusion as one of the main objectives of the examination. Perhaps it would be also timely to consider an additional assessment parameter of quantifying the quality of smile attractiveness to supplement the quality of achieved occlusion since the delivery of orthodontic treatment involves both the scientific knowledge that governs orthodontic diagnostic and biomechanical skill set and the artistic eyes that frame and design the perceived smile attractiveness. The assessment of smile attractiveness could include parameters such as the symmetry of the smile, smile index, smile line ratio, smile arc, upper lip line, upper lip curvature, and buccal corridor. ${ }^{32}$ The evaluation of smile attractiveness could be applied as a bonus to differentiate candidates with excellent and comprehensive orthodontic skill set from those who deliver passable and average treatment outcomes in the pursuit of orthodontic excellence ( - Fig. 3 ).

The importance of examining beyond the finished occlusion from study models is needful in contemporary orthodontics. The major focus of assessing orthodontic treatment outcomes has traditionally been the use of occlusal indices that primarily focus on orthodontic treatment need and outcomes based on intra-arch and interarch occlusal traits and relationships. The Dental Aesthetic Index was developed to score the malocclusion severity and justify orthodontic

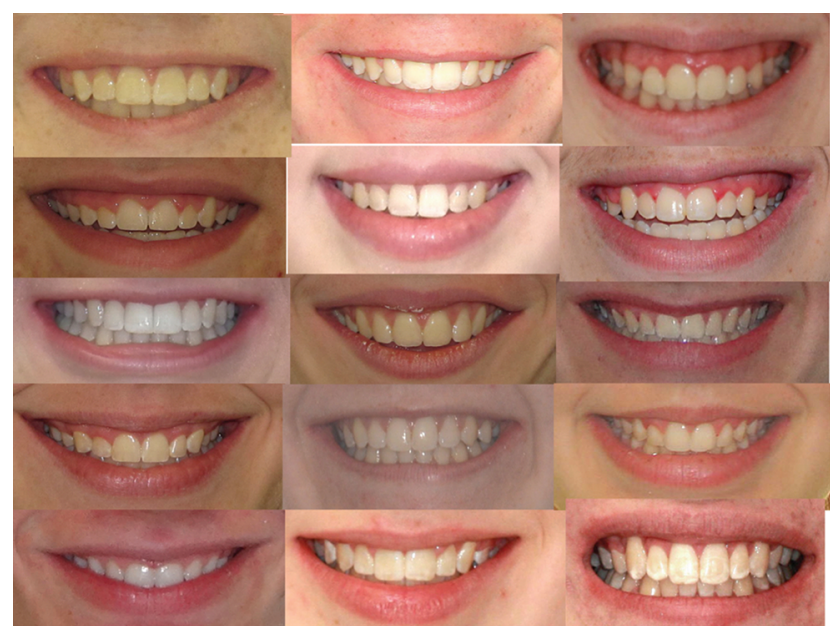

Fig. 3 Sample of smile photographs used in the study. 
treatment need based on occlusal traits that potentially compromised dental esthetics with inherent limitations when smile attractiveness is to be evaluated in totality based on the findings of the present study. ${ }^{33}$ The aesthetic component of the Index of Orthodontic Treatment Need gives emphasis to dental spacing, dental crowding, overjet, and overbite evaluated from 10 photographs of static dentitions in occlusion. ${ }^{34}$ Other features of the dentition such as tooth morphology, tooth color and the interplay among the teeth, gingiva, and lips on smiling have not been taken into consideration in the assessment of the quality of treatment outcomes with the use of occlusal indices that were primarily designed for epidemiological and funding purposes. The use of study model for the evaluation of smile attractiveness has its limitations beyond the clinical parameters color smile photographs of the anterior occlusion can offer.

The present and previously published studies also highlighted the lack of congruence in view of a perfect smile was not unique to any particular community but a common dilemma faced by both Caucasian and non-Caucasian health professionals. $^{29}$ The predictors of smile attractiveness in descending order of influence were the gums (1.82; $95 \%$ confidence interval $[\mathrm{CI}]: 1.92,1.73)$, the teeth $(1.56 ; 95 \% \mathrm{CI}: 1.66$, 1.47), and the lips (1.47; 95\% CI: $1.56,1.37)$. These results suggest that the role of the gums such as plaque-induced iatrogenic gingival hyperplasia associated with fixed orthodontic appliances or naturally occurring excessive gingival show associated with vertical maxillary excess and/or high lip line on smiling could affect the perceived quality of smile attractiveness more so than the teeth and the lips. These findings could infer and reiterate the importance of good oral hygiene practices during orthodontic treatment is essential to prevent plaque-induced gingivitis and white spot lesions which are the most common adverse effects associated with orthodontic treatment. ${ }^{35,36}$ White spot lesions cause discoloration of the enamel of teeth that inevitably affects the perceived smile attractiveness that could possibly impact perceived judgment of social competence and appeal, intellectual ability and relationship satisfaction. ${ }^{16}$ It is also interesting to note that the straightness of teeth, something orthodontist strive so hard to achieve, is also affected by the gums, anatomy of the teeth, and lip architecture. These factors are out of the hands of orthodontists alone and are important factors to consider in the greater multidisciplinary team of cosmetic dentistry and facial aesthetics. Disease or abnormalities will add to the aesthetic outcomes of a perceived attractive outcome.

Plastic surgeons were found to be more critical than orthodontists when assessing smile attractiveness. Perhaps the training background of plastic surgeons with a strong emphasis on soft tissue had influenced their assessment of the smile attractiveness in relation to the lips and smile line relative to the teeth and gingiva. Further investigation as to how plastic surgeons perceive and assess smile attractiveness would be needful and especially helpful in the collaborative management of cleft lip and palate patients. There were no significant predictors found among periodontists, prosthodontists, and orthodontists in the assessment of smile attractiveness. This finding could mean that the dental professionals involved in the present study had similar viewpoint when assessing smile attractiveness. It could be due to the commonality in the dental training program with the evaluation of smile attractiveness. It would be crucial to have a common understanding of smile attractiveness among dental professionals when managing multidisciplinary cases. As such, a holistic approach toward orthodontic treatment planning should be emphasized with participation from various relevant experts from other dental specialties to deliver the best possible treatment outcome tailored to the specific dental needs of an individual orthodontic patient. $^{37}$

The ROC curve generated a cutoff point of 7.4 as the rating score that defined what was assessed to be an attractive smile in perfect harmony with the teeth, gums, and lips. Only 2 of the 68 cases had rating scores above 7.4 suggesting that the majority of the cases that satisfied the ABO-OGS standards did not display all the traits that contributed to the perfect smile attractiveness. This finding reiterated the idea put forth that a good static occlusion does not necessarily translate to an attractive smile as the established teeth position with orthodontic treatment should not be considered in isolation but in totality and holistically with tooth alignment, overjet, overbite, tooth morphology, tooth color, gingiva, and lips when smiling being reviewed as a single entity.

The present study found that gender of the non-Caucasian health professionals was not associated with the assessors' ratings irrespective of the clinical discipline of the health professionals. The assessors who participated in the present study had an age range between 20 and 40 years. Thus, the association between age of assessors and ratings was not investigated as the age range was too narrow to establish meaningful comparison.

\section{Limitations}

The investigators of the present study consciously made the decision to use colored photographs to demonstrate the role of the gums and tooth color on smile attractiveness. A limitation was the use of colored photographs taken at the time of the removal of fixed orthodontic appliances for some patients and did not invite the patients to return to clinic to be assessed again after the reduction of the gingival inflammation caused by the orthodontic appliances. The limited time available to conduct the study resulted in the decision to use all available photographs disregarding the state of gingival inflammation that would likely improve over time.

Some previously published studies suggest that the use of photographs was not a reliable way to study smile attractiveness as this method could not capture the dynamic smile unlike the video recording method. ${ }^{38,39}$ However, in the best interest of time, it was not possible to invite each of the 68 consecutive patients back to perform video recordings. As such, the investigators of the present study decided to use photographs instead with the knowledge of the compromise made. 
The other limitation was the difference in subsample sizes among the four difference clinical disciplines, in particular, the prosthodontist and plastic surgeon subgroup. The use of larger and comparable subsample sizes of various clinical disciplines is recommended for future studies.

Finally, the study did not incorporate a group of lay people which is also a limitation of the study. Specialists have been shown in previous studies to be more critical to the clinical outcomes of the smile aesthetics. ${ }^{22,40,41}$ However, in the modern day, with patient access to social media and the Internet, the expectation of the layperson is becoming more and more. Clinicians will need to understand their role in the realms of aesthetic dentistry and outcomes.

\section{Conclusions}

The findings from the study suggested the following conclusions:

1. Only 2 out of 68 ABO certified cases were considered to have attained an attractive smile based on ROC cutoff rating score of 7.4.

2. Plastic surgeons were more critical in the assessment of smile attractiveness compared with orthodontists.

3. No difference was found among orthodontists, periodontists, and prosthodontists in the assessment of smile attractiveness.

4. Gender was not a predictor of smile attractiveness ratings.

5. The gums, teeth, and lips were associated with smile attractiveness in the descending order of influence.

\section{Conflict of Interest}

None declared.

\section{Acknowledgments}

The authors wish to acknowledge Dr Tayem Abou Saleh for the work on pictures and questionnaire, and Dr Rong Bing Xie for statistical analysis.

\section{References}

1 Lin F, Ren M, Yao L, He Y, Guo J, Ye Q. Psychosocial impact of dental esthetics regulates motivation to seek orthodontic treatment. Am J Orthod Dentofacial Orthop 2016;150(3):476-482

2 Pithon MM, Nascimento CC, Barbosa GC, Coqueiro RdaS. Do dental esthetics have any influence on finding a job? Am J Orthod Dentofacial Orthop 2014;146(4):423-429

3 Khela S, Newton JT, Jeremiah HG. The effect of malocclusion on dating prospects. J Orthod 2020;47(1):30-37

4 Dos Santos PR, Meneghim MC, Ambrosano GM, Filho MV, Vedovello SA. Influence of quality of life, self-perception, and self-esteem on orthodontic treatment need. Am J Orthod Dentofacial Orthop 2017;151(1):143-147

5 OlsenJA, Inglehart MR. Malocclusions and perceptions of attractiveness, intelligence, and personality, and behavioral intentions. Am J Orthod Dentofacial Orthop 2011;140(5):669-679

6 Christou T, Abarca R, Christou V, Kau CH. Smile outcome comparison of Invisalign and traditional fixed-appliance treatment: a case-control study. Am J Orthod Dentofacial Orthop 2020;157(3):357-364

7 Christou T, Betlej A, Aswad N, Ogdon D, Kau CH. Clinical effectiveness of orthodontic treatment on smile esthetics: a systematic review. Clin Cosmet Investig Dent 2019;11:89-101
8 Sadowsky SJ. An overview of treatment considerations for esthetic restorations: a review of the literature. J Prosthet Dent 2006;96(6):433-442

9 Sarver DM. The importance of incisor positioning in the esthetic smile: the smile arc. Am J Orthod Dentofacial Orthop 2001;120(2):98-111

10 Sarver DM, Ackerman MB. Dynamic smile visualization and quantification: part 2. Smile analysis and treatment strategies. Am J Orthod Dentofacial Orthop 2003;124(2):116-127

11 Sarver DM, Ackerman MB. Dynamic smile visualization and quantification: part 1. Evolution of the concept and dynamic records for smile capture. Am J Orthod Dentofacial Orthop 2003;124(1):4-12

12 Nold SL, Horvath SD, Stampf S, Blatz MB. Analysis of select facial and dental esthetic parameters. Int J Periodontics Restorative Dent 2014;34(5):623-629

13 Cao L, Zhang K, Bai D, Jing Y, Tian Y, Guo Y. Effect of maxillary incisor labiolingual inclination and anteroposterior position on smiling profile esthetics. Angle Orthod 2011;81(1):121-129

14 Heravi F, Rashed R, Abachizadeh H. Esthetic preferences for the shape of anterior teeth in a posed smile. Am J Orthod Dentofacial Orthop 2011;139(6):806-814

15 Ong E, Brown RA, Richmond S. Peer assessment of dental attractiveness. AmJOrthod Dentofacial Orthop 2006;130(2):163-169

16 Joiner A, Luo W. Tooth colour and whiteness: a review. J Dent 2017;67S:S3-S10

17 GoodacreCJ.Gingivalesthetics.JProsthetDent1990;64(1):1-12

18 Kokich VO, Kokich VG, Kiyak HA. Perceptions of dental professionals and laypersons to altered dental esthetics: asymmetric and symmetric situations. Am J Orthod Dentofacial Orthop 2006;130(2):141-151

19 Oliveira PLE, Motta A, Pithon M, Mucha J. Details of pleasing smiles. Int J Esthet Dent 2018;13(4):494-514

20 Wang C, Hu WJ, Liang LZ, Zhang YL, Chung KH. Esthetics and smile-related characteristics assessed by laypersons. J Esthet Restor Dent 2018;30(2):136-145

21 Scott CR, Goonewardene MS, Murray K. Influence of lips on the perception of malocclusion. Am J Orthod Dentofacial Orthop 2006;130(2):152-162

22 Parrini S, Rossini G, Castroflorio T, Fortini A, Deregibus A, Debernardi C. Laypeople's perceptions of frontal smile esthetics: a systematic review. Am J Orthod Dentofacial Orthop 2016; 150(5):740-750

23 Pinho S, Ciriaco C, Faber J, Lenza MA. Impact of dental asymmetries on the perception of smile esthetics. Am J Orthod Dentofacial Orthop 2007;132(6):748-753

24 Soh J, Chew MT, Chan YH. Perceptions of dental esthetics of Asian orthodontists and laypersons. Am J Orthod Dentofacial Orthop 2006;130(2):170-176

25 Richmond S, Shaw WC, Roberts CT, Andrews M. The PAR index (Peer Assessment Rating): methods to determine outcome of orthodontic treatment in terms of improvement and standards. Eur J Orthod 1992;14(3):180-187

26 Daniels C, Richmond S. The development of the index of complexity, outcome and need (ICON). J Orthod 2000;27(2):149-162

27 Akyalcin S, Frels LK, English JD, Laman S. Analysis of smile esthetics in American Board of Orthodontic patients. Angle Orthod 2014;84(3):486-491

28 Schabel BJ, McNamara JA, Baccetti T, Franchi L, Jamieson SA. The relationship between posttreatment smile esthetics and the ABO Objective Grading System. Angle Orthod 2008;78(4):579-584

29 Kau CH, Christou T, Xie RB, Abou-Saleh T. Rating of smile attractiveness of patients finished to the American Board of Orthodontics standards. J Orofac Orthop 2020;81(4): 239-248

30 Swets JA. Indices of discrimination or diagnostic accuracy: their ROCs and implied models. Psychol Bull 1986;99(1):100-117 
31 Swets JA. ROC analysis applied to the evaluation of medical imaging techniques. Invest Radiol 1979;14(2):109-121

32 Durgekar SG, K N, Naik V. The ideal smile and its orthodontic implications. World J Orthod 2010;11(3):211-220

33 Jenny J, Cons NC. Establishing malocclusion severity levels on the Dental Aesthetic Index (DAI) scale. Aust Dent J 1996;41(1):43-46

34 Brook PH, Shaw WC. The development of an index of orthodontic treatment priority. Eur J Orthod 1989;11(3):309-320

35 Albhaisi Z, Al-Khateeb SN, Abu Alhaija ES. Enamel demineralization during clear aligner orthodontic treatment compared with fixed appliance therapy, evaluated with quantitative light-induced fluorescence: a randomized clinical trial. Am J Orthod Dentofacial Orthop 2020;157(5):594-601

36 Verrusio C, Iorio-Siciliano V, Blasi A, Leuci S, Adamo D, Nicolò $M$. The effect of orthodontic treatment on periodontal tissue inflammation: a systematic review. Quintessence Int 2018;49(1):69-77
37 Abdelkarim A, Jerrold L. Strategies for improved interdisciplinary care and communication in orthodontics. Am J Orthod Dentofacial Orthop 2017;152(5):717-721

38 Walder JF, Freeman K, Lipp MJ, Nicolay OF, Cisneros GJ. Photographic and videographic assessment of the smile: objective and subjective evaluations of posed and spontaneous smiles. Am J Orthod Dentofacial Orthop 2013;144(6):793-801

39 Mahn E, Sampaio CS, Pereira da Silva B, et al. Comparing the use of static versus dynamic images to evaluate a smile. J Prosthet Dent 2020;123(5):739-746

40 Al Taki A, Khalesi M, Shagmani M, Yahia I, Al Kaddah F. Perceptions of altered smile esthetics: a comparative evaluation in orthodontists, dentists, and laypersons. Int J Dent 2016;2016:7815274

41 Kokich VO Jr, Kiyak HA, Shapiro PA. Comparing the perception of dentists and lay people to altered dental esthetics. J Esthet Dent 1999;11(6):311-324 